\title{
Rapid Detection of Pesticide Residues in Chinese Herbal Medicines by Fourier Transform Infrared Spectroscopy Coupled with Partial Least Squares Regression
}

\author{
Tianming Yang, Rong Zhou, Du Jiang, Haiyan Fu, Rui Su, Yangxi Liu, and Hanbo Su \\ School of Pharmaceutical Sciences, South-Central University for Nationalities, Wuhan 430074, China \\ Correspondence should be addressed to Haiyan Fu; fuhaiyan@mail.scuec.edu.cn
}

Received 2 August 2016; Revised 5 October 2016; Accepted 14 November 2016

Academic Editor: Khalique Ahmed

Copyright (c) 2016 Tianming Yang et al. This is an open access article distributed under the Creative Commons Attribution License, which permits unrestricted use, distribution, and reproduction in any medium, provided the original work is properly cited.

\begin{abstract}
This paper reports a simple, rapid, and effective method for simultaneous detection of cartap (Ca), thiocyclam (Th), and tebufenozide (Te) in Chinese herbal medicines including Radix Angelicae Dahuricae and Liquorices using Fourier transform infrared spectroscopy (FT-IR) coupled with partial least squares regression (PLSR). The proposed method can handle the intrinsic interferences of herbal samples; satisfactory average recoveries attained from near-infrared (NIR) and mid-infrared (MIR) PLSR models were $99.0 \pm 10.8$ and $100.2 \pm 1.0 \%$ for $\mathrm{Ca}, 100.2 \pm 6.9$ and $99.7 \pm 2.5 \%$ for Th, and $99.1 \pm 6.3$ and $99.6 \pm 1.0 \%$ for Te, respectively. Furthermore, some statistical parameters and figures of merit are fully investigated to evaluate the performance of the two models. It was found that both models could give accurate results and only the performance of MIR-PLSR was slightly better than that of NIR-PLSR in the cases suffering from herbal matrix interferences. In conclusion, FT-IR spectroscopy in combination with PLSR has been demonstrated for its application in rapid screening and quantitative analysis of multipesticide residues in Chinese herbal medicines without physical or chemical separation pretreatment step and any spectral processing, which also implies other potential applications such as food and drug safety, herbal plants quality, and environmental evaluation, due to its advantages of nontoxic and nondestructive analysis.
\end{abstract}

\section{Introduction}

Chinese herbal medicines (CHMs) which were considered to be gentle, nontoxic, and even harmless have been widely used as a means of medication or dietary supplement $[1,2]$. In order to prevent, repel, or mitigate the effects of pest, the commercial cultivation of CHMs receives frequent application of diverse pesticides which are highly effective and broadspectrum but have long half-life, complex degradation, and highly toxic substances. Consequently, the widespread use of pesticides poses high risks to the environment and induces heavy adverse effects on human health [3-6]. Facing such a serious crisis, for safety and health, it is therefore important to establish an effective routine method for quantifying multipesticide residues in Chinese herbal medicines.

Based on the available literature, a number of analytical methods have been proposed for the determination of pesticide residues in different matrices [7-12], including gas chromatography (GC), gas chromatography-mass spectrometry (GC-MS), high performance liquid chromatography (HPLC), and ultrahigh performance liquid chromatographymass spectrometry (UPLC-MS). Unfortunately, these techniques are time-consuming and reagent-demanding and require highly skilled operators. Therefore, a faster, more accurate, and sensitive identification method is urgent to be developed for its practical application. In recent years, Fourier transform near-infrared (NIR) and mid-infrared (MIR) spectroscopies with advantages such as high efficiency, low cost, simply measuring, little sample preparation, quick data analysis, and nondestructive analytical technique have been widely used in several scientific fields, such as medical and biomedical, food science, pharmaceutical, and petroleum industries [13-17]. However, it should be noticed that only a few papers have described analytical approaches for monitoring multipesticide residues in Chinese herbal medicines by using FT-IR. This is due to the fact that fingerprint 
information of multipesticide residues in Chinese herbal provided by NIR and MIR spectra may be difficult to directly interpret due to low resolution and overlapping peak bands, so effective and robust chemometrics methods have been extensively concerned to extract and relate the abundant IR spectra information [18-20].

The main role of chemometrics for quantitative analysis of NIR and MIR data is to establish a quantitative model relating the measured NIR signals to certain properties of samples, say the component content. The quantitative model was subsequently applied to predict the same properties of samples in the prediction set. So, a good prediction result relies a good multivariate calibration method. Many methods such as principal component regression (PCR) [21], multiple linear regression (MLR) [22], and partial least squares (PLS) [23] are often used for chemometric calibration. In particular, partial least squares regression (PLSR), as a well-performed multivariate data analysis technique which generalizes and combines features from principal component analysis and multiple linear regression, is especially suitable for modeling from a full spectrum [24]. It is a procedure used to relate a large number of independent variables (predictors) to one (PLSR1) or few (PLSR2) response variables (observations) when a reduced number of cases are available and is useful in predicting a set of dependent variables from a large set of independent collinear variables. Since it reduces a great amount of redundant information, PLSR is ideal for multivariate calibration of spectroscopic data $[25,26]$. In recent years, attention was paid to the application of PLSR in various disciplines and a large number of studies reported successful results [27-29].

In the present paper, a rapid and effective strategy for simultaneous determination of cartap (Ca), thiocyclam (Th), and tebufenozide (Te) in CHMs including Radix Angelicae Dahuricae and Liquorices has been proposed, by combining FI-IR with PLSR algorithm. The results have revealed that the direct determination of pesticide residues in complicated CHMs can be achieved, which adequately exploits the simple, rapid, and accuracy advantage with no requirement of physical or chemical separation and spectral processing, indicating a promising quantitative alternative for CHMs quality control and online monitoring of pesticide residues. Moreover, the accuracy and figures of merit of both NIRPLSR and MIR-PLSR methods, including average recoveries, root mean square error, and limit of detection (LOD), were investigated and compared to evaluate the performance of the developed methods.

\section{Materials and Methods}

2.1. Apparatus. A NICOLET 6700 FT-IR, OMNIC 8.2 spectral collecting software (Thermo Fisher Scientific Inc., USA), Antaris II FT-NIR spectrometer, and RESULT 3.0 spectral collecting software (Thermo Electron Co., USA) were used.

\subsection{Reagents and Samples. Radix Angelicae Dahuricae and} Liquorices samples were obtained from Gansu in China. Cartap, thiocyclam, and tebufenozide were collected from Agro-Environmental Protection Institute of the Ministry of Agriculture (Tianjin, China). Chromatography-grade carbinol was purchased from TEDIA (TEDIA, USA). $\mathrm{KBr}$ (99.8\% purity) was purchased from Aladdin Industrial Corporation (Shanghai, China).

2.3. Sample Preparation. The purchased standard solutions $\left(100 \mu \mathrm{g} \cdot \mathrm{mL}^{-1}\right)$ of individual pesticides were stored in dark glass vials at $-20^{\circ} \mathrm{C}$. The standard solutions were further diluted with carbinol to prepare the working solutions for calibration and verification in recovery studies. Calibration standards were $8 \mu \mathrm{g} \cdot \mathrm{mL}^{-1}$. All solutions were stored in dark glass vials at $4^{\circ} \mathrm{C}$.

Radix Angelicae Dahuricae and Liquorices samples used in NIR and MIR were crushed with the grinder and sifted into fine powders by 200 mesh sieve and then vacuumdried at $60^{\circ} \mathrm{C}$ for 24 hours. Each Radix Angelicae Dahuricae or Liquorices powdered sample was weighed accurately (about $1.0 \mathrm{~g}$ ). Different volumes of the working solutions of pesticide were further added into each CHMs sample and completely mixed into the prepared samples. The Radix Angelicae Dahuricae and Liquorices samples were divided into seven groups (R1-R7) and (L1-L7), respectively. That is, in each of the 7 groups (R1-R7) and (L1-L7), 30 samples with different Radix Angelicae Dahuricae and Liquorices matrices were prepared, respectively, and then vacuum-dried at $60^{\circ} \mathrm{C}$ for 24 hours. The sieved powders were stored in a dryer spare. Detailed information about the added content of the working pesticide solutions was listed in Table 1.

2.4. Spectra Acquisition. MIR spectra were recorded using $\mathrm{KBr}$ pellets and the wavenumber ranged from 4000 to $400 \mathrm{~cm}^{-1}$ with a resolution of $4 \mathrm{~cm}^{-1}$ and 30 samples with different herbal matrices in each group was collected, 30 spectra every day. NIR spectra were acquired by the diffuse reflectance mode; the spectral range was from 10000 to $4000 \mathrm{~cm}^{-1}$ with a resolution of $8 \mathrm{~cm}^{-1}$ and 30 samples with different herbal matrices in each group was collected, 30 spectra every day. The average spectra of three parallel measured spectra for each sample in each group were adopted to construct model. The same operation was repeated for three consecutive days.

2.5. Method of Chemometrics. PLSR programs were written and performed using a Matlab 2010a (Math Works, Natick, MA, USA).

Partial least squares regression (PLSR) was used for developing quantitative NIR and MIR models of cartap (Ca), thiocyclam (Th), and tebufenozide (Te) in CHMs including Radix Angelicae Dahuricae and Liquorices. In this work, considering $n \times p$ matrix $\mathbf{X}$ including $p$ predictor variables (NIR or MIR spectral variables) for $n$ CHMs samples and $n \times 1$ vector including the corresponding dependent variable for $n$ CHMs samples, for simplicity and without loss of generality, both $\mathbf{X}$ and $\mathbf{y}$ are column centered; the goal of PLS is to find a set of orthogonal latent variables that are the linear combinations of the original predictor variables. The dependent variable is subsequently regressed against the latent variables. Because a calibration model with high 
TABLE 1: Concentrations of $\mathrm{Ca}$, Th, and Te in CHMs samples.

\begin{tabular}{|c|c|c|c|c|}
\hline \multirow{2}{*}{$\mathrm{CHMs}$} & \multirow{2}{*}{ Sample groups } & \multicolumn{3}{|c|}{ Added concentration $\left(\mu \mathrm{g} \cdot \mathrm{g}^{-1}\right)$} \\
\hline & & $\mathrm{Ca}$ & Th & $\mathrm{Te}$ \\
\hline \multirow{7}{*}{ Radix Angelicae Dahuricae } & $\mathrm{R} 1$ & 40.00 & 0.00 & 0.00 \\
\hline & $\mathrm{R} 2$ & 32.00 & 8.00 & 0.00 \\
\hline & $\mathrm{R} 3$ & 24.00 & 12.00 & 4.00 \\
\hline & $\mathrm{R} 4$ & 16.00 & 16.00 & 8.00 \\
\hline & R5 & 8.00 & 20.00 & 12.00 \\
\hline & R6 & 0.00 & 24.00 & 16.00 \\
\hline & R7 & 8.00 & 12.00 & 20.00 \\
\hline \multirow{7}{*}{ Liquorices } & L1 & 40.00 & 0.00 & 0.00 \\
\hline & $\mathrm{L} 2$ & 32.00 & 8.00 & 0.00 \\
\hline & L3 & 24.00 & 12.00 & 4.00 \\
\hline & $\mathrm{L} 4$ & 16.00 & 16.00 & 8.00 \\
\hline & L5 & 8.00 & 20.00 & 12.00 \\
\hline & L6 & 0.00 & 24.00 & 16.00 \\
\hline & L7 & 8.00 & 12.00 & 20.00 \\
\hline
\end{tabular}

TABLE 2: A detail calibration and validation CHMs samples of PLSR models based on NIR and MIR.

\begin{tabular}{|c|c|c|c|c|}
\hline \multirow{2}{*}{ Sample groups } & \multicolumn{2}{|c|}{ NIR-PLSR } & \multicolumn{2}{|c|}{ MIR-PLSR } \\
\hline & Training number & Prediction number & Training number & Prediction number \\
\hline R1 & 22 & 8 & 18 & 12 \\
\hline $\mathrm{R} 2$ & 19 & 11 & 14 & 16 \\
\hline R3 & 19 & 11 & 19 & 11 \\
\hline $\mathrm{R} 4$ & 19 & 11 & 16 & 14 \\
\hline $\mathrm{R} 5$ & 21 & 9 & 22 & 8 \\
\hline R6 & 17 & 13 & 17 & 13 \\
\hline R7 & 18 & 12 & 21 & 9 \\
\hline L1 & 23 & 7 & 18 & 12 \\
\hline $\mathrm{L} 2$ & 22 & 8 & 14 & 16 \\
\hline L3 & 20 & 10 & 19 & 11 \\
\hline $\mathrm{L} 4$ & 23 & 7 & 16 & 14 \\
\hline L5 & 17 & 13 & 22 & 8 \\
\hline L6 & 21 & 9 & 17 & 13 \\
\hline L7 & 19 & 11 & 21 & 9 \\
\hline
\end{tabular}

complexity tends to give degraded prediction performance and has a higher risk of overfitting, it is important to make a proper tradeoff between model complexity and accuracy. Therefore, different models with the optimum latent variables (Lvs) were investigated by $n$-fold cross-validation.

2.6. Method Validation. The 210 NIR spectra data of Radix Angelicae Dahuricae or Liquorices samples were randomly divided into two sets: 135 spectra were used for calibration and 75 spectra were used for validation. The 210 MIR spectra data of Radix Angelicae Dahuricae or Liquorices samples were randomly divided into two sets: 127 spectra were used for calibration and 83 spectra were used for validation of constructed PLSR models; detailed sample information was listed in Table 2.

Root mean square error of 8 -fold cross-validation (RMSECV) was used as an index to select the number of latent variables. The correlation coefficient $(r)$, regression equation, LOD, LOQ, average recoveries, and intraday and interday precisions were used to validate this method. Precision and repeatability were determined by calculating intraday and interday relative standard deviation of calibration (RSDC) and prediction (RSDP) to evaluate the performance of instrument. The limit of detection (LOD) $[30,31]$ and the limit of quantification (LOQ) are computed as follows:

$$
\begin{aligned}
& \mathrm{LOD}=3.3 s(0) \\
& \mathrm{LOQ}=10 s(0),
\end{aligned}
$$

where $s(0)$ is the standard deviation in the predicted concentration for herbal sample background blank samples.

Performance of the models was estimated in terms of calibration root-mean-squared error (RMSEC), prediction root-mean-squared error (RMSEP) with the optimal latent 


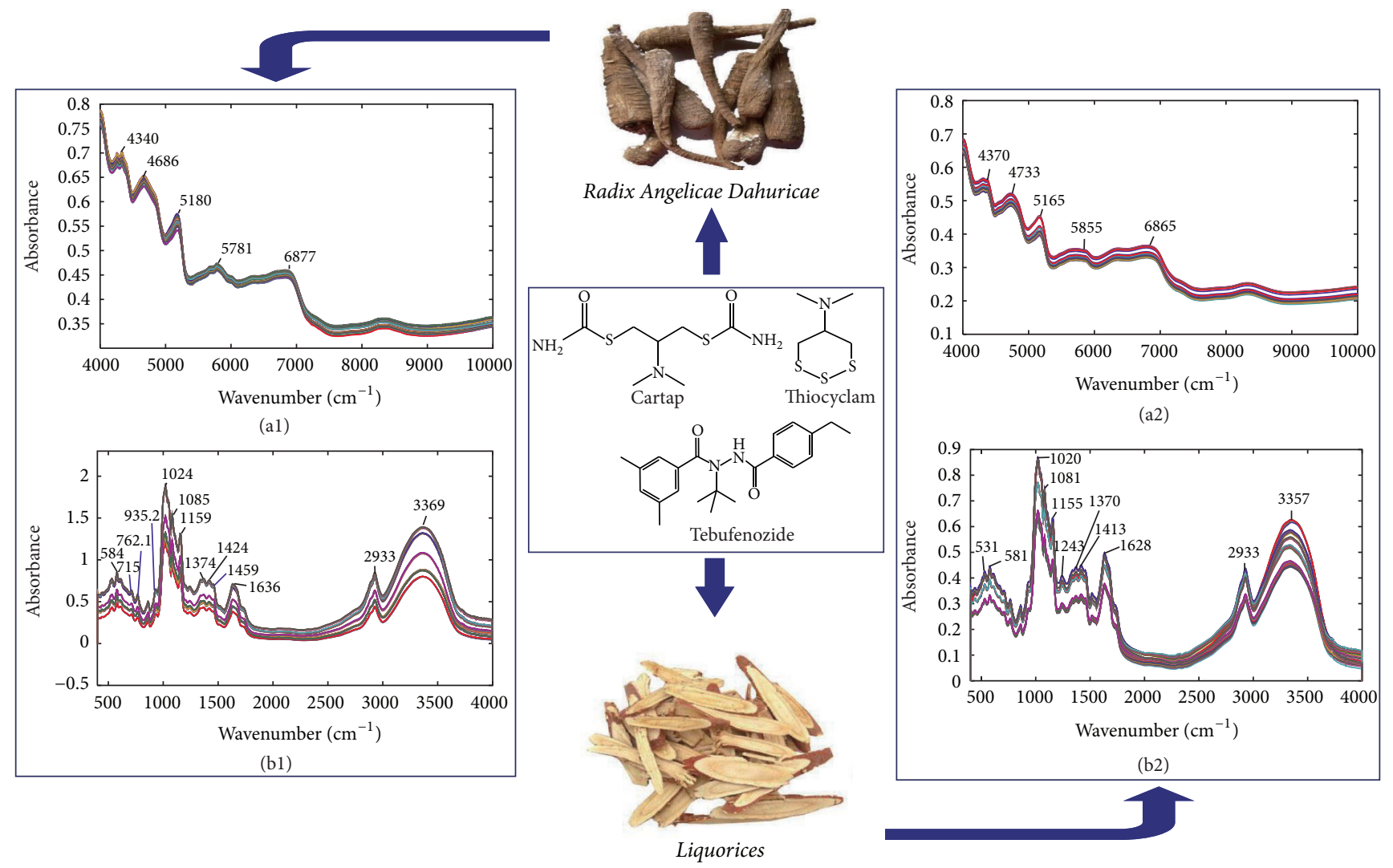

FIGURE 1: The molecular structures of cartap, thiocyclam, and tebufenozide and the original NIR ((a1) and (a2)) or MIR ((b1) and (b2)) spectra of multipesticide residues in Radix Angelicae Dahuricae and Liquorices samples.

variable, and the correlation coefficient between the reference and the predicted values as the following equations [32]:

$$
\begin{aligned}
\text { RMSEC } & =\sqrt{\frac{\sum_{i=1}\left(y_{c i}-\hat{y}_{c i}\right)^{2}}{N c}} \\
\text { RMSEP } & =\sqrt{\frac{\sum_{i=1}\left(y_{p i}-\hat{y}_{p i}\right)^{2}}{N p}} \\
R & =\frac{\sum_{i=1}\left(y_{i}-\hat{y}_{i}\right)^{2}}{\sqrt{s^{2}\left(y_{i}\right) \times s^{2}\left(\hat{y}_{i}\right)}},
\end{aligned}
$$

where $N c$ and $N p$ correspond to the number of calibration samples and validation samples, respectively. $y_{c i}$ and $\hat{y}_{c i}$ are the references and predicted values of the property $y$ for $i$ th calibration sample, respectively. $y_{p i}$ and $\hat{y}_{p i}$ represent the reference and predicted values of the property $y$ for $i$ th validation sample. $s^{2}\left(y_{i}\right)$ and $s^{2}\left(\hat{y}_{i}\right)$ denote the variances for the reference and predicted values for the interesting component $y_{i}$, respectively.

\section{Results and Discussions}

3.1. Analysis of NIR and MIR Spectral Fingerprints of Multipesticide Residues in CHMs Samples. The molecular structures of three pesticide residues including $\mathrm{Ca}$, $\mathrm{Th}$, and $\mathrm{Te}$ and the NIR and MIR spectra of multipesticide residues in Radix Angelicae Dahuricae and Liquorices samples are plotted in Figure 1.

The raw NIR or MIR spectra are highly overlapped and have a poor peak resolution, which makes the accurate assignments of specific peaks very difficult. For ease of peak attributions, chemical bonds are denoted as atomatom, where an atom can be carbon $(\mathrm{C})$, hydrogen $(\mathrm{H})$, oxygen $(\mathrm{O})$, and nitrogen $(\mathrm{N})$. For the NIR spectra of multipesticide residues in Radix Angelicae Dahuricae samples as shown in Figure 1(a1), it can be seen that the characteristic absorption peaks can be interpreted as follows $[33,34]$ : the peak $4251 \mathrm{~cm}^{-1}$ is the $\mathrm{C}-\mathrm{H}$ stretch/C-H deformation in the phenyl or $\mathrm{CH}_{2}$ bend second overtone and around $4340 \mathrm{~cm}^{-1}$ can be attributed to the combination absorbance of C- $\mathrm{H}$ antisymmetric stretching and C-H bending. Around $4686 \mathrm{~cm}^{-1}$ peak brand is due to the combination stretching vibration of $\mathrm{C}=\mathrm{C},=\mathrm{C}-\mathrm{H}$ bands and combination of the base bands of $\mathrm{N}$ $\mathrm{H}$ stretching and bending; peak $5180 \mathrm{~cm}^{-1}$ can be explained as second overtone of $\mathrm{C}=\mathrm{O}$ stretching bands, stretching first overtone of $\mathrm{C}-\mathrm{H}$ bands in aromatic rings and combination of the basebands of $\mathrm{O}-\mathrm{H}$ stretching and bending. Other perk assignments $5781 \mathrm{~cm}^{-1}$ as the second overtones of C$\mathrm{H}$ stretching in various groups and $6877 \mathrm{~cm}^{-1}$ as the first overtone of $\mathrm{O}-\mathrm{H}$ stretching. Figure 1(b1) showed MIR spectra 
of multipesticide residues in Radix Angelicae Dahuricae samples. Seen from Figure 1(b1), variation around the peak at $1200-900 \mathrm{~cm}^{-1}$ can be associated with C-H group and the peak at $1500-1200 \mathrm{~cm}^{-1}$ can be associated with C-O. The wide scope between 3500 and $1700 \mathrm{~cm}^{-1}$ mainly consists of the overlapping of -OH stretching $\left(3500-2933 \mathrm{~cm}^{-1}\right)$ and various -NH bending and stretching vibrations of amide compounds $\left(3400-1636 \mathrm{~cm}^{-1}\right)$. Other peaks might be assigned as asymmetric vibrations of $\mathrm{CH}_{2}$ at $2933 \mathrm{~cm}^{-1}$ and characteristic key bands around $715 \mathrm{~cm}^{-1}$ and $762.1 \mathrm{~cm}^{-1}$ can be regarded as fine features of aromatic substitution. In general, different functional groups of the multipesticide residues or chemical constituents in Radix Angelicae Dahuricae such as isoimperatorin, imperatorin, oxypeucedanin, byakangelicol, and byakangelicin could be assigned to different vibration modes. The low spectral resolution can be attributed to the contributions of multicomponents and the shifts and distortions resulted from their interactions. Similarly, NIR and MIR spectral fingerprints of multipesticide residues in Liquorices also can reflect characteristic of chemical bonds and still suffered from low spectral resolution and overlapping. Therefore, chemometric methods are required to extract useful information for simultaneous quantitative analysis of $\mathrm{Ca}$, Th, and Te in CHMs.

3.2. Simultaneous Determination of $\mathrm{Ca}$, Th, and $\mathrm{Te}$ in $\mathrm{CHMs}$ Samples. For simultaneous quantitative analysis of $\mathrm{Ca}$, Th, and Te in CHMs samples, linear PLS models were developed to relate the raw FT-NIR spectra or FT-MIR to multipesticide residues of Radix Angelicae Dahuricae and Liquorices, respectively. The optimum latent variables numbers of all PLSR models were determined as 6 by 8 -fold cross-validation. The corresponding predicted concentration to the analyte (every pesticide residue) as a function with its actual concentration can be found to evaluate relation and deviations through a linear regression equation. Herein, variable $y$ was correlated with the prediction value of pesticide residues, while variable $x$ was correlated with the actual value of pesticide residues. The correlation coefficients of $\mathrm{Ca}$, Th, and $\mathrm{Te}$ obtained by using both NIR-PLSR and MIR-PLSR is nearly close to 1 , respectively. The prediction results for the different CHMs using the NIR and MIR methods based on PLSR are summarized in Table 3.

For $\mathrm{Ca}$, Th, and Te in Liquorices, the average predicted recoveries gained from NIR are $99.0 \pm 10.8,100.2 \pm 6.9$, and $99.1 \pm 6.3 \%$ and from MIR are 100.2 $\pm 1.0,99.7 \pm 2.5$, and $99.6 \pm 1.0 \%$, respectively. For $\mathrm{Ca}$, Th, and Te in Radix Angelicae Dahuricae, the average predicted recoveries gained from NIR are 100.4 $\pm 6.1,98.8 \pm 6.6$, and $100.4 \pm 4.6 \%$ and from MIR are $100.0 \pm 0.1,99.7 \pm 2.8$, and $100.0 \pm$ $1.1 \%$, respectively. These results show that both methods can provide a satisfactory prediction capacity to potentially determine $\mathrm{Ca}$, Th, and Te in complicated CHMs matrices. In addition, figures of merit (FOM) such as LOD and LOQ are very important in developing, comparing, and assessing the reliability of analytical methodologies and analytical results. For NIR-PLSR method, the limits of detection (LODs) for $\mathrm{Ca}, \mathrm{Th}$, and Te in Radix Angelicae Dahuricae are 2.45, 1.20, and $1.15 \mu \mathrm{g} \cdot \mathrm{g}^{-1}$, respectively; in Liquorices are 2.03, 1.75, and $2.36 \mu \mathrm{g} \cdot \mathrm{g}^{-1}$, respectively. For MIR-PLSR method, LODs for $\mathrm{Ca}, \mathrm{Th}$, and Te in Radix Angelicae Dahuricae are 0.40, 0.27, and $0.14 \mathrm{ng} \cdot \mathrm{mL}^{-1}$, respectively; in Liquorices are $0.38,0.32$, and $0.30 \mu \mathrm{g} \cdot \mathrm{g}^{-1}$. One can find that the proposed NIR and MIR method based on PLSR can yield satisfactory predictive capacity for determination of ATR, AME, and PRO in Radix Angelicae Dahuricae and Liquorices samples, respectively. The predicted concentrations versus the actual ones for $\mathrm{Ca}(\mathrm{a}), \mathrm{Th}$ (b), and Te (c) in Radix Angelicae Dahuricae using NIR-PLSR without spectra preprocessing were also showed in Figure 2. It is observed that prediction values were very close to actual value. Similarly, prediction value of the three pesticide residues in Radix Angelicae Dahuricae and Liquorices by NIRPLSR or MIR-PLSR is very slightly deviated from actual value (similar figures were not shown). These results confirmed that the NIR method based on PLSR was fairly effective.

Moreover, for the sake of a further investigation into the accuracy and of the proposed methods, 30 Radix Angelicae Dahuricae samples of each group from seven different groups (R1-R7) and 30 Liquorices samples of each group from seven different groups (L1-L7) were prepared and analyzed in triplicate in a day; this assay was repeated for 3 days. Statistical parameters including RMSEC, RMSEP, RSDC, and RSDP in intraday and interday testing are demonstrated in Table 4.

It is observed that NIR-PLSR gives intraday RMSEP as $0.59,0.79$, and 0.43 and interday RMSEP as 1.54, 1.14, and 0.67 for Ca, Th, and Te in Radix Angelicae Dahuricae, respectively. MIR-PLSR gives intraday RMSEP as $0.11,0.39$, and 0.08 and interday RMSEP as $0.57,0.53$, and 0.69 for $\mathrm{Ca}$, Th, and Te in Radix Angelicae Dahuricae, respectively. It can be also seen that all the relative standard deviations (RSDPs) were less than $10 \%$ by NIR-PLSR and $7 \%$ by MIR-PLSR. These results further verify that the proposed two methods could give accurate results as an alternative to each other; only the performance of MIR-PLSR was slightly better than that of NIR-PLSR under the circumstances suffering from matrix effects of CHMs. This might be due to the fact that the MIR shows the strongest absorption in the infrared active vibration.

\section{Conclusion}

In the present study, a simple, rapid, and effective method has been successfully developed for quantitative analysis of $\mathrm{Ca}, \mathrm{Th}$, and Te in Radix Angelicae Dahuricae and Liquorices based on the PLSR using both FT-NIR and FT-MIR methods. The proposed quantitative method proved to be capable of performing the simultaneous determination of multipesticide residues in complex Chinese herbal medicines without physical or chemical separation pretreatment step and spectral processing. Furthermore, the figures of merit and statistical parameters indicated that both methods could give accurate and stable results; only the performance of MIRPLSR was slightly better than that of NIR-PLSR in the cases suffering from matrix effects. It is expected that the potential advantages of this determination of trace pesticide in Chinese herbal medicines, such as accuracy, rapidity, and low cost, can 


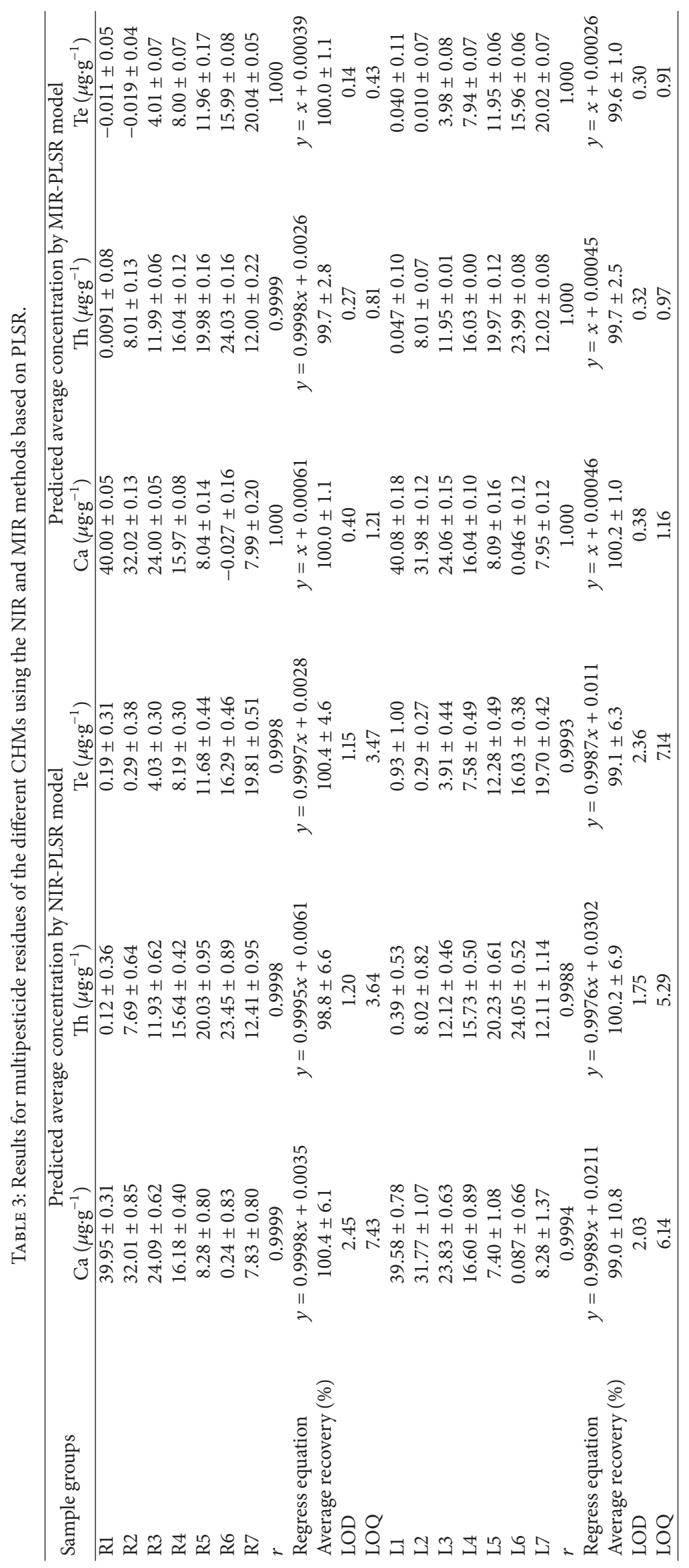


TABLE 4: The results obtained for validation assays of accuracy and precision in CHMs samples.

\begin{tabular}{|c|c|c|c|c|c|c|c|c|c|c|}
\hline \multirow{3}{*}{ Methods } & \multirow{3}{*}{ CHMs } & \multirow{3}{*}{ Pesticides } & \multicolumn{4}{|c|}{ Intraday } & \multicolumn{4}{|c|}{ Interday } \\
\hline & & & \multicolumn{2}{|c|}{ Accuracy } & \multicolumn{2}{|c|}{ Precision } & \multicolumn{2}{|c|}{ Accuracy } & \multicolumn{2}{|c|}{ Precision } \\
\hline & & & RMSEC & RMSEP & RSDC $\%$ & RSDP\% & RMSEC & RMSEP & RSDC\% & RSDP\% \\
\hline \multirow{4}{*}{ NIR-PLSR } & \multirow{6}{*}{ Radix Angelicae Dahuricae } & $\mathrm{Ca}$ & 0.48 & 0.59 & 2.09 & 2.55 & 1.12 & 1.54 & 4.66 & 7.30 \\
\hline & & Th & 0.47 & 0.79 & 2.83 & 4.69 & 0.76 & 1.14 & 4.49 & 6.74 \\
\hline & & $\mathrm{Te}$ & 0.33 & 0.43 & 3.18 & 3.94 & 0.47 & 0.67 & 4.30 & 6.37 \\
\hline & & $\mathrm{Ca}$ & 0.08 & 0.11 & 0.36 & 0.42 & 0.48 & 0.57 & 2.26 & 2.41 \\
\hline \multirow{2}{*}{ MIR-PLSR } & & Th & 0.10 & 0.39 & 0.61 & 1.06 & 0.33 & 0.53 & 1.92 & 3.09 \\
\hline & & $\mathrm{Te}$ & 0.05 & 0.08 & 0.43 & 0.77 & 0.50 & 0.69 & 4.71 & 6.80 \\
\hline \multirow{4}{*}{ NIR-PLSR } & & $\mathrm{Ca}$ & 0.45 & 1.01 & 1.90 & 5.00 & 1.50 & 1.96 & 6.40 & 9.20 \\
\hline & & Th & 0.36 & 0.85 & 2.30 & 4.20 & 0.86 & 1.24 & 5.30 & 7.00 \\
\hline & & $\mathrm{Te}$ & 0.26 & 0.55 & 2.60 & 4.60 & 0.67 & 0.85 & 6.40 & 6.60 \\
\hline & Liquorices & $\mathrm{Ca}$ & 0.067 & 0.14 & 0.30 & 0.60 & 0.38 & 0.46 & 1.60 & 1.80 \\
\hline \multirow{2}{*}{ MIR-PLSR } & & Th & 0.042 & 0.34 & 0.20 & 0.80 & 0.34 & 0.43 & 1.90 & 2.80 \\
\hline & & $\mathrm{Te}$ & 0.038 & 0.082 & 0.30 & 0.80 & 0.22 & 0.31 & 1.90 & 3.30 \\
\hline
\end{tabular}

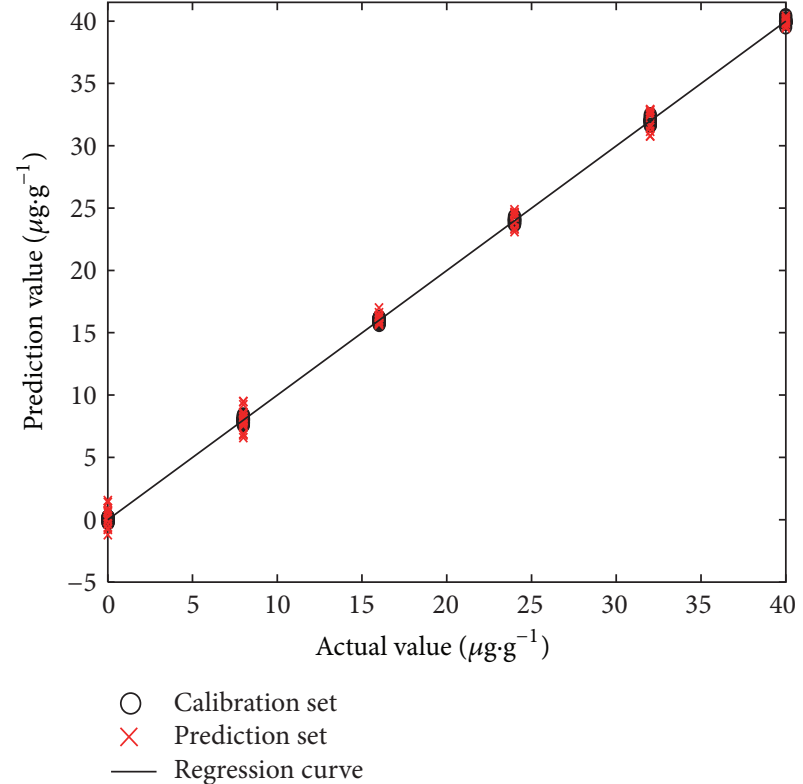

(a)

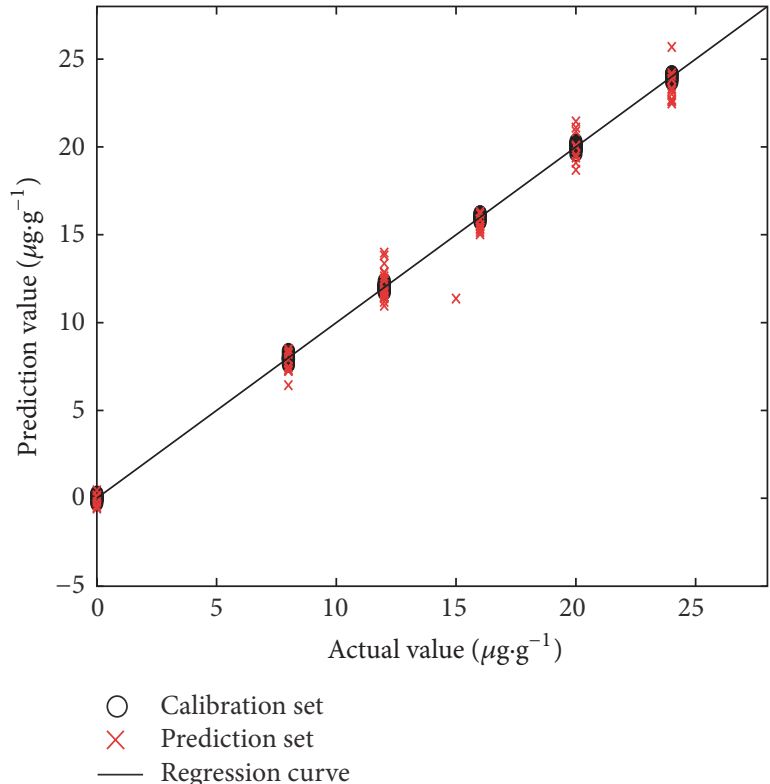

(b)

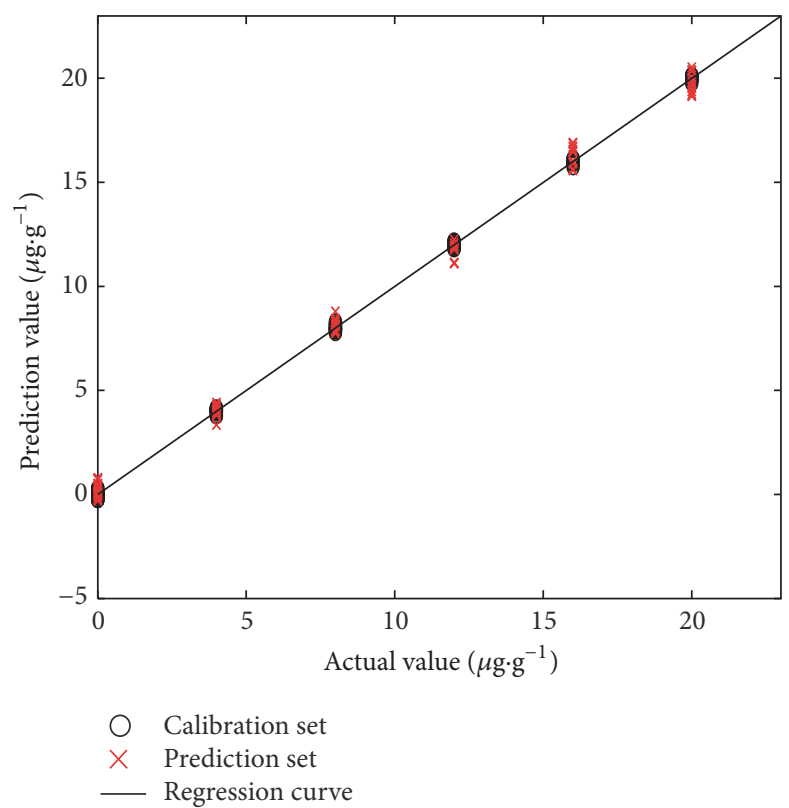

(c)

FIGURE 2: Correlation plots of the predicted concentrations versus the actual ones for Ca (a), Th (b), and Te (c) in Radix Angelicae Dahuricae using NIR-PLSR. 
be even more highlighted by considering the possibility of automating the proposed methods for online detection.

\section{Competing Interests}

The authors declare that they have no competing interests.

\section{Acknowledgments}

This work was financially supported by the National Natural Science Foundation of China [nos. 21576297 and 21205145]; the Open Funds of State Key Laboratory Breeding Base of Green Chemistry-Synthesis Technology of Zhejiang University of Technology [no. GCTKF2014003]; the Open Research Program [nos. 2015ZD001 and 2015ZD002] from the Modernization Engineering Technology Research Center of Ethnic Minority Medicine of Hubei province (South-Central University for Nationalities).

\section{References}

[1] K. Chan, "Some aspects of toxic contaminants in herbal medicines," Chemosphere, vol. 52, no. 9, pp. 1361-1371, 2003.

[2] C. W. Huie, "A review of modern sample-preparation techniques for the extraction and analysis of medicinal plants," Analytical and Bioanalytical Chemistry, vol. 373, no. 1-2, pp. 2330, 2002.

[3] K. Banerjee, S. Utture, S. Dasgupta et al., "Multiresidue determination of 375 organic contaminants including pesticides, polychlorinated biphenyls and polyaromatic hydrocarbons in fruits and vegetables by gas chromatography-triple quadrupole mass spectrometry with introduction of semi-quantification approach," Journal of Chromatography A, vol. 1270, pp. 283-295, 2012.

[4] Z. H. Rivera, E. Oosterink, L. Rietveld, F. Schoutsen, and L. Stolker, "Influence of natural organic matter on the screening of pharmaceuticals in water by using liquid chromatography with full scan mass spectrometry," Analytica Chimica Acta, vol. 700, no. 1-2, pp. 114-125, 2011.

[5] A. Garcia-Ac, P. A. Segura, L. Viglino et al., "On-line solidphase extraction of large-volume injections coupled to liquid chromatography-tandem mass spectrometry for the quantitation and confirmation of 14 selected trace organic contaminants in drinking and surface water," Journal of Chromatography A, vol. 1216, no. 48, pp. 8518-8527, 2009.

[6] F. Hernández, T. Portolés, E. Pitarch, and F. J. López, "Target and nontarget screening of organic micropollutants in water by solid-phase microextraction combined with gas chromatography/high-resolution time-of-flight mass spectrometry," Analytical Chemistry, vol. 79, no. 24, pp. 9494-9504, 2007.

[7] Q. Gou, X. Lv, L. Tan, and B.-Y. Yu, "Simultaneous determination of 26 pesticide residues in 5 Chinese medicinal materials using solid-phase extraction and GC-ECD method," Chinese Journal of Natural Medicines, vol. 7, no. 3, pp. 210-216, 2009.

[8] R. Xu, J. Wu, Y. Liu et al., "Analysis of pesticide residues using the Quick Easy Cheap Effective Rugged and Safe (QuEChERS) pesticide multiresidue method in traditional Chinese medicine by gas chromatography with electron capture detection," Chemosphere, vol. 84, no. 7, pp. 908-912, 2011.
[9] H. Tong, Y. Tong, J. Xue, D. Liu, and X. Wu, "Multi-residual pesticide monitoring in commercial chinese herbal medicines by gas chromatography-triple quadrupole tandem mass spectrometry," Food Analytical Methods, vol. 7, no. 1, pp. 135-145, 2014.

[10] X. Mao, Y. Wan, A. Yan, M. Shen, and Y. Wei, "Simultaneous determination of organophosphorus, organochlorine, pyrethriod and carbamate pesticides in Radix astragali by microwave-assisted extraction/dispersive-solid phase extraction coupled with GC-MS," Talanta, vol. 97, pp. 131-141, 2012.

[11] B. Guo, S. Ji, F. Zhang, B. Yang, J. Gu, and X. Liang, "Preparation of $\mathrm{C}_{18}$-functionalized $\mathrm{Fe}_{3} \mathrm{O}_{4} @ \mathrm{SiO}_{2}$ core-shell magnetic nanoparticles for extraction and determination of phthalic acid esters in Chinese herb preparations," Journal of Pharmaceutical and Biomedical Analysis, vol. 100, pp. 365-368, 2014.

[12] L. Chen, F. Song, Z. Liu, Z. Zheng, J. Xing, and S. Liu, "Multiresidue method for fast determination of pesticide residues in plants used in traditional chinese medicine by ultra-highperformance liquid chromatography coupled to tandem mass spectrometry," Journal of Chromatography A, vol. 1225, pp. 132140, 2012.

[13] R. M. Balabin and R. Z. Safieva, "Near-infrared (NIR) spectroscopy for biodiesel analysis: fractional composition, iodine value, and cold filter plugging point from one vibrational spectrum," Energy \& Fuels, vol. 25, no. 5, pp. 2373-2382, 2011.

[14] A. P. Craig, A. S. Franca, L. S. Oliveira, J. Irudayaraj, and K. Ileleji, "Application of elastic net and infrared spectroscopy in the discrimination between defective and non-defective roasted coffees," Talanta, vol. 128, pp. 393-400, 2014.

[15] Y. Feng, D. Lei, and C. Hu, "Rapid identification of illegal synthetic adulterants in herbal anti-diabetic medicines using near infrared spectroscopy," Spectrochimica Acta-Part A: Molecular and Biomolecular Spectroscopy, vol. 125, pp. 363-374, 2014.

[16] J. Peerapattana, K. Otsuka, and M. Otsuka, "Application of NIR spectroscopy for the quality control of mangosteen pericarp powder: quantitative analysis of alpha-mangostin in mangosteen pericarp powder and capsule," Journal of Natural Medicines, vol. 67, no. 3, pp. 452-459, 2013.

[17] C. Zhang and J. Su, "Application of near infrared spectroscopy to the analysis and fast quality assessment of traditional Chinese medicinal products," Acta Pharmaceutica Sinica B, vol. 4, no. 3, pp. 182-192, 2014.

[18] J. J. Liu, H. Xu, W. S. Cai, and X. G. Shao, "Discrimination of industrial products by on-line near infrared spectroscopy with an improved dendrogram," Chinese Chemical Letters, vol. 22, no. 10, pp. 1241-1244, 2011.

[19] O. Galtier, O. Abbas, Y. Le Dréau et al., "Comparison of PLS1DA, PLS2-DA and SIMCA for classification by origin of crude petroleum oils by MIR and virgin olive oils by NIR for different spectral regions," Vibrational Spectroscopy, vol. 55, no. 1, pp. 132140, 2011.

[20] W. Q. Luo, S. Y. Huan, H. Y. Fu et al., "Preliminary study on the application of near infrared spectroscopy and pattern recognition methods to classify different types of apple samples," Food Chemistry, vol. 128, no. 2, pp. 555-561, 2011.

[21] P. Geladi and B. R. Kowalski, "Partial least-squares regression: a tutorial," Analytica Chimica Acta, vol. 185, pp. 1-17, 1986.

[22] Q. Shen, J.-H. Jiang, C.-X. Jiao, G.-L. Shen, and R.-Q. Yu, "Modified particle swarm optimization algorithm for variable selection in MLR and PLS modeling: QSAR studies of antagonism of angiotensin II antagonists," European Journal of Pharmaceutical Sciences, vol. 22, no. 2-3, pp. 145-152, 2004. 
[23] H. Kubinyi, "Evolutionary variable selection in regression and PLS analyses," Journal of Chemometrics, vol. 10, no. 2, pp. 119133, 1996.

[24] M. Mirzaie, R. Darvishzadeh, A. Shakiba, A. A. Matkan, C. Atzberger, and A. Skidmore, "Comparative analysis of different uni- and multi-variate methods for estimation of vegetation water content using hyper-spectral measurements," International Journal of Applied Earth Observation and Geoinformation, vol. 26, no. 1, pp. 1-11, 2014.

[25] P. Lin, Y. Chen, and Y. He, "Identification of geographical origin of olive oil using visible and near-infrared spectroscopy technique combined with chemometrics," Food and Bioprocess Technology, vol. 5, no. 1, pp. 235-242, 2012.

[26] M. M. Pojić and J. S. Mastilović, "Near infrared spectroscopyadvanced analytical tool in wheat breeding, trade, and processing," Food and Bioprocess Technology, vol. 6, no. 2, pp. 330-352, 2013.

[27] L. Ragni, A. Berardinelli, C. Cevoli, and E. Valli, "Assessment of the water content in extra virgin olive oils by Time Domain Reflectometry (TDR) and Partial Least Squares (PLS) regression methods," Journal of Food Engineering, vol. 111, no. 1, pp. 66-72, 2012.

[28] I. Gouvinhas, J. M. M. M. de Almeida, T. Carvalho, N. Machado, and A. I. R. N. A. Barros, "Discrimination and characterisation of extra virgin olive oils from three cultivars in different maturation stages using Fourier transform infrared spectroscopy in tandem with chemometrics," Food Chemistry, vol. 174, no. 4, pp. 226-232, 2015.

[29] S. A. Moreira, J. Sarraguça, D. F. Saraiva, R. Carvalho, and J. A. Lopes, "Optimization of NIR spectroscopy based PLSR models for critical properties of vegetable oils used in biodiesel production," Fuel, vol. 150, pp. 697-704, 2015.

[30] A. Lorber, K. Fabert, and B. R. Kowalski, "Net analyte signal calculation in multivariate calibration," Analytical Chemistry, vol. 69, no. 8, pp. 1620-1626, 1997.

[31] M. J. Rodríguez-Cuesta, R. Boqué, F. X. Rius, J. L. M. Vidal, and A. G. Frenich, "Development and validation of a method for determining pesticides in groundwater from complex overlapped HPLC signals and multivariate curve resolution," Chemometrics and Intelligent Laboratory Systems, vol. 77, no. 1-2, pp. 251-260, 2005.

[32] A. M. K. Pedro and M. M. C. Ferreira, "Nondestructive determination of solids and carotenoids in tomato products by near-infrared spectroscopy and multivariate calibration," Analytical Chemistry, vol. 77, no. 8, pp. 2505-2511, 2005.

[33] B. M. Nicolaï, K. Beullens, E. Bobelyn et al., "Nondestructive measurement of fruit and vegetable quality by means of NIR spectroscopy: a review," Postharvest Biology and Technology, vol. 46, no. 2, pp. 99-118, 2007.

[34] B. C. Tang, H. Y. Fu, Q. B. Yin et al., "Combining near-infrared spectroscopy and chemometrics for rapid recognition of an $\mathrm{Hg}$ contaminated plant," Journal of Spectroscopy, vol. 2016, Article ID 3597451, 7 pages, 2016. 

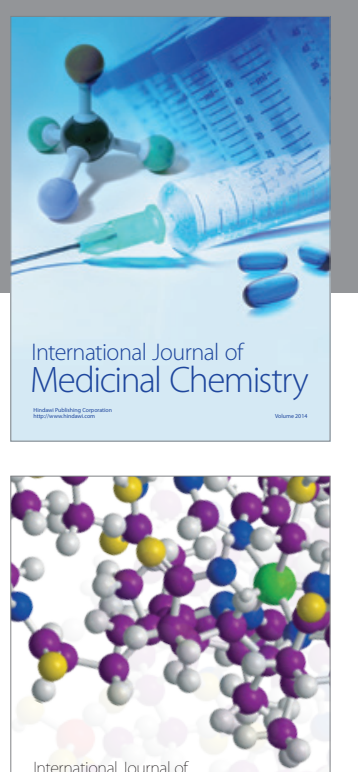

Carbohydrate Chemistry

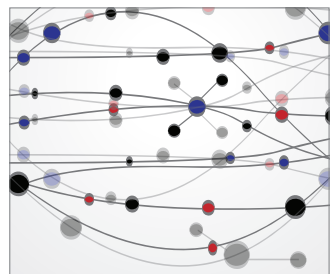

The Scientific World Journal
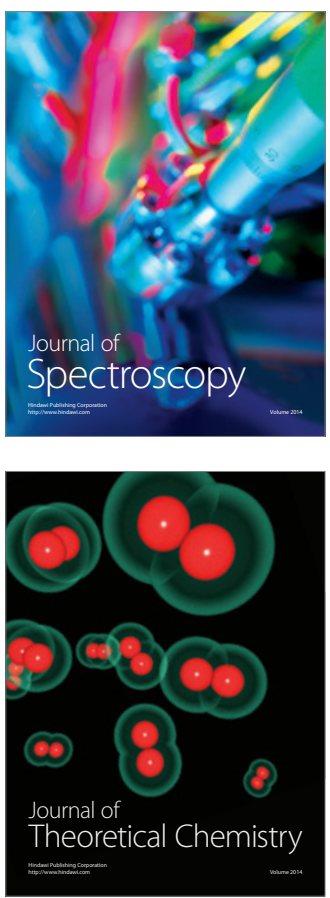
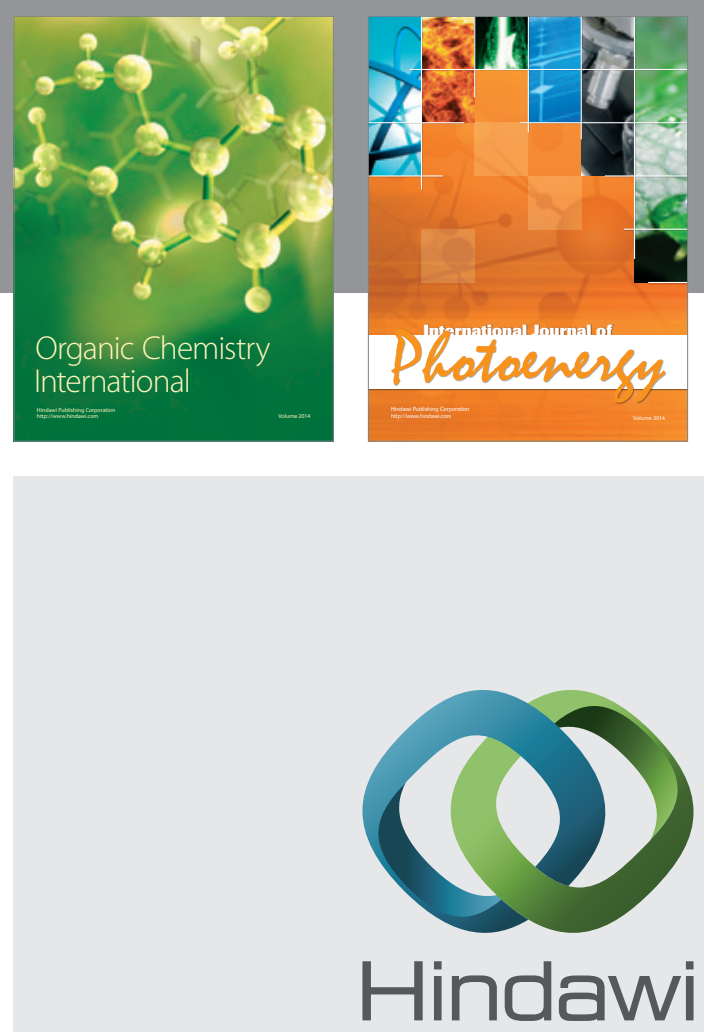

Submit your manuscripts at

http://www.hindawi.com

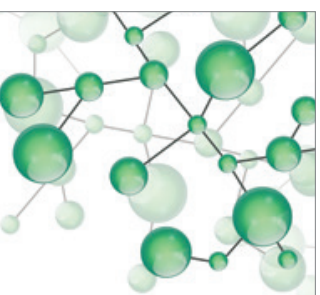

International Journal of

Inorganic Chemistry

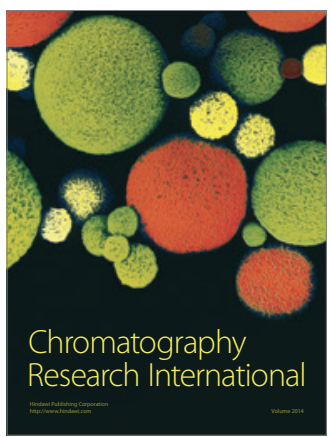

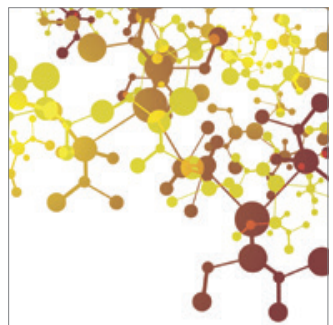

Applied Chemistry
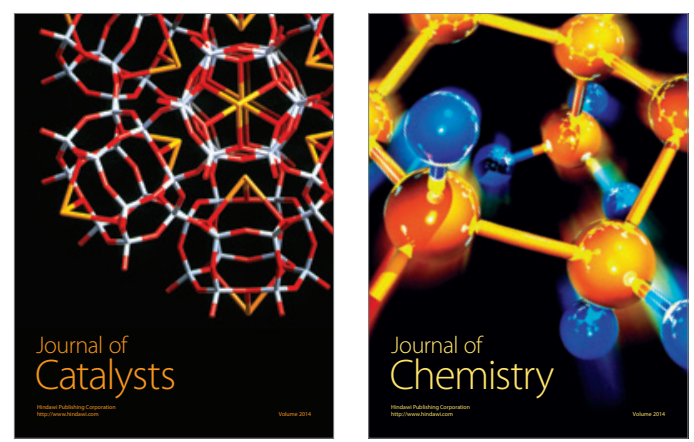
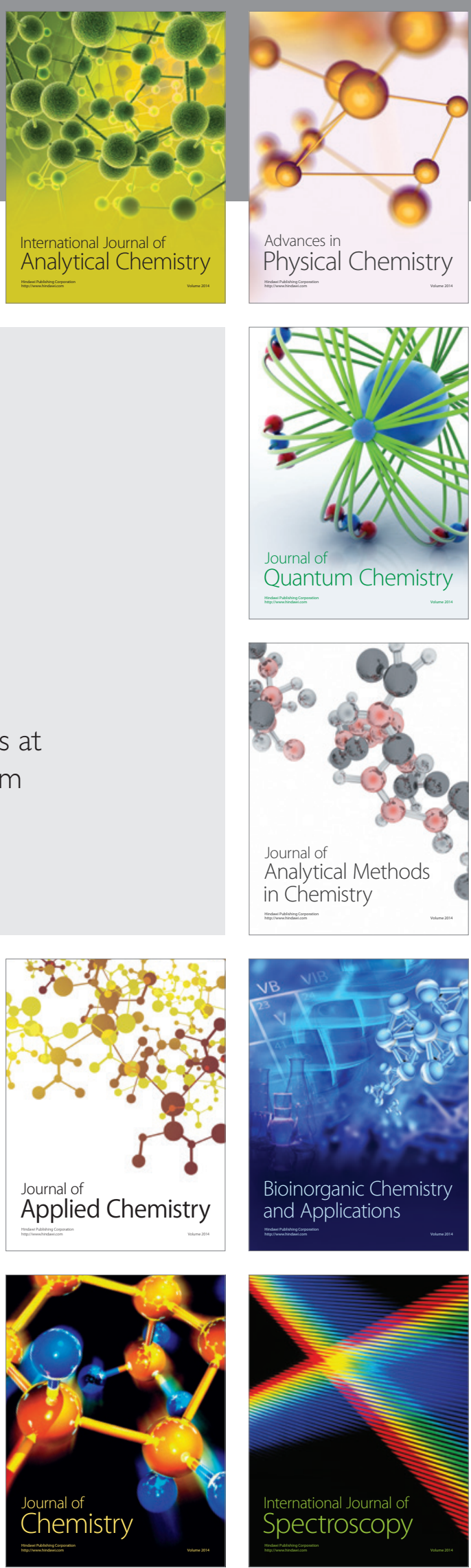